external granular layer for lateral desmoplastic tumors. Research involving the signal transduction mechanisms that promote oncogenesis includes sonic hedgehog (Shh), wingless (Wnt) growth factor receptors, receptor tyrosine kinases, retinoids that induce apoptosis, and notch and CXCR4 signalling ligands and inhibitors of apoptosis proteins that are associated with a poor prognosis. Determining the mechanisms of signal transduction in neurooncogenesis and the genetics of medulloblastoma will aid the development of more specifically targeted therapies. (Crawford JR, MacDonald TJ, Packer RJ. Medulloblastoma in childhood: new biological advances. Lancet Neurol December 2007;6:1073-1085). (Respond: John R Crawford MD, Children's National Medical Center, The George Washington University, 111 Michigan Ave NW, Washington DC 20010).

COMMENT. The addition of chemotherapy in the past 2-3 decades has increased the survival rate compared to surgical resection and radiotherapy alone. Controversial methods of management include total tumor resection that might account for the increased incidence of posterior fossa mutism; whole body radiation that causes long-term intellectual deterioration; and the incorporation of biological therapeutics in combination with radiation or chemotherapy. Altered classification of tumors based on more advanced neuroimaging may explain an apparent improvement in survival rates in response to new treatments.

\title{
OUTCOME OF INFANT EXTRACEREBRAL FLUID COLLECTIONS
}

Cases of benign infantile extracerebral fluid collections and nontraumatic chronic subdural hygroma or hematoma were reviewed retrospectively at Nebraska Medical Center, Omaha. Thirty-nine patients with benign extracerebral fluid collections presented at 3-12 months of age with macrocephaly. The birth was premature in 15 cases. Head size was normal at birth, 3 required shunt placement, but in most the accelerated head growth leveled off, approaching the $98^{\text {th }}$ percentile after 24 months of age. Long-term developmental outcome was normal in 30 and mildly delayed in 3.

Of 9 patients with subdural hygroma, 3 presented with a large head and 3 with seizures. Birth was premature in 4. Five required subdural punctures and/or subduroperitoneal shunt placement. All developed normally. (Hellbusch LC. Benign extracerebral fluid collections in infancy: clinical presentation and long-term follow-up. Jrnl Neurosurgery: Pediatrics 2007;107(2):119-125). (Respond: Dr Leslie C Hellbusch, Department of Surgery (Neurosurgery), Nebraska Medical Center, Omaha, Nebraska).

COMMENT. In this patient series, infants with benign extracerebral fluid collections had a favorable long-term outcome, and $44 \%$ of patients with nontraumatic subdural hygroma resolved without surgical intervention. Previous follow-up studies of infants with idiopathic macrocephaly and orbito-frontal extradural fluid collections have shown variable results regarding neurological, radiological, and neuropsychological outcome. Some showed normalization of head circumference by 18-24 months, and others had persistent radiological changes. A study of 41 infants wih idiopathic macrocephaly and extradural fluid collections followed to young adulthood showed resolution of the fluid collection but neuropsychological impairments in visuomotor skills and attention. (Muenchberger $\mathrm{H}$ et al. Child's Nerv Syst 2006;22(10):1242-1248; Ped Neur Briefs Nov 2006;20:81-82). 